\title{
THE QUASAR SDSS J105041.35+345631.3: BLACK HOLE RECOIL OR EXTREME DOUBLE-PEAKED EMITTER?
}

\author{
G. A. Shields ${ }^{1}$, D. J. Rosario ${ }^{2}$, K. L. Smith ${ }^{1}$, E. W. Bonning ${ }^{3}$, S. Salviander ${ }^{1}$, J. S. Kalirai ${ }^{4}$, R. Strickler ${ }^{2}$, \\ E. Ramirez-Ruiz ${ }^{2}$, A. A. Dutton ${ }^{2}$, T. Treu ${ }^{5}$, and P. J. Marshall ${ }^{5}$ \\ ${ }^{1}$ Department of Astronomy, University of Texas, Austin, TX 78712, USA; shields@astro.as.utexas.edu, krista@ @ail.utexas.edu, triples@astro.as.utexas.edu \\ ${ }^{2}$ Lick Observatory, University of California, Santa Cruz, CA 95064, USA; rosario@ucolick.org, rrs@ ucolick.org, enrico@ucolick.org, dutton@ucolick.org \\ ${ }^{3}$ YCAA - Department of Physics, Yale University, New Haven, CT 06520, USA; erin.bonning@ @ale.edu \\ ${ }^{4}$ Space Telescope Science Institute, 3700 San Martin Drive, Baltimore, MD 21218, USA; jkalirai@ stsci.edu \\ ${ }^{5}$ Department of Physics, University of California, Santa Barbara, CA 93106, USA; tt@ physics.ucsb.edu, pjm @ physics.ucsb.edu \\ Received 2009 July 20; accepted 2009 October 29; published 2009 November 30
}

\begin{abstract}
The quasar SDSS J105041.35+345631.3 $(z=0.272)$ has broad emission lines blueshifted by $3500 \mathrm{~km} \mathrm{~s}^{-1}$ relative to the narrow lines and the host galaxy. Such an object may be a candidate for a recoiling supermassive black hole, a binary black hole, a superposition of two objects, or an unusual geometry for the broad emission-line region. The absence of narrow lines at the broad line redshift argues against superposition. New Keck spectra of $\mathrm{J} 1050+3456$ place tight constraints on the binary model. The combination of large velocity shift and symmetrical $\mathrm{H} \beta$ profile, as well as aspects of the narrow line spectrum, make $\mathrm{J} 1050+3456$ an interesting candidate for black hole recoil. Other aspects of the spectrum, however, suggest that the object is most likely an extreme case of a "doublepeaked emitter." We discuss possible observational tests to determine the true nature of this exceptional object.
\end{abstract}

Key words: black hole physics - galaxies: active - quasars: general

Online-only material: color figure

\section{INTRODUCTION}

Mergers of binary black holes can result in the final black hole receiving a "kick" due to anisotropic emission of gravitational radiation at coalescence. Kick velocities are predicted to range up to a maximum of $4000 \mathrm{~km} \mathrm{~s}^{-1}$ for certain configurations of black hole spin and mass ratio (Campanelli et al. 2007). For the case of supermassive black holes merging in a galaxy with sufficient gas to power an accretion disk around the black hole, the merger remnant will carry a portion of the disk with it and be visible as a quasar, either physically offset from the nucleus or with broad emission lines Doppler-shifted from the host galaxy velocity (Loeb 2007; Madau \& Quataert 2004; Blecha \& Loeb 2008). Detection of a recoiling black hole would be a powerful confirmation of the predictions of numerical relativity and shed light on the cosmic evolution of supermassive black holes. Spectroscopic searches for kicked quasars have had limited success. Bonning et al. (2007) reported upper limits to the incidence of kicks at various velocities in a sample of spectra from the Sloan Digital Sky Survey. ${ }^{6}$ One quasar, SDSS J092712.65+294344.0, has been proposed by Komossa et al. (2008) as a recoil candidate on the basis of its spectrum; however, other physical models for this object have been proposed (Bogdanović et al. 2009; Dotti et al. 2009; Heckman et al. 2009; Shields et al. 2009). Another object, SDSS $\mathrm{J} 153636.22+044127.0$, was proposed as a sub-parsec binary supermassive black hole on the basis of a large blueshift of its broad emission lines (Boroson \& Lauer 2008). Subsequent observations (Chornock et al. 2009; Lauer \& Boroson 2009) indicate that it is a member of the "double-peaked emitter" class of active galactic nuclei (AGNs), as discussed below.

The peculiar spectrum of $\mathrm{J} 1050+3456$ was noticed in the course of a search for binary quasi-stellar object (QSO) candidates involving visual inspection of $\sim 21,000$ spectra of QSOs

\footnotetext{
6 The SDSS Web site is http://www.sdss.org.
}

at $0.1<z<0.7$ from SDSS Data Release 5 (Smith et al. 2009). The object shows narrow lines with typical AGN line ratios, at a redshift consistent with the stellar absorption lines. The broad $\mathrm{H} \beta$ line has a symmetrical profile blueshifted by $3500 \mathrm{~km} \mathrm{~s}^{-1}$, near the upper end of the range predicted for gravitational radiation recoil. In view of the importance of discovering examples of recoil in nature, all candidate objects merit close attention. On the other hand, as emphasized by Bonning et al. (2007) and Chornock et al. (2009), most cases of displaced broad lines will be double-peaked emitters rather than kicks or binaries. Here, we present new spectroscopic observations of $\mathrm{J} 1050+3456$ and discuss alternative models for its physical nature, including the possibility that it is an extreme case of a double peaked emitter. We assume a concordance cosmology with $\mathrm{H}_{0}=70 \mathrm{~km} \mathrm{~s}^{-1} \mathrm{Mpc}^{-1}, \Omega_{\Lambda}=0.7$. Spectral features are labeled using air wavelengths.

\section{SPECTRAL PROPERTIES OF J1050+3456}

\subsection{The SDSS Spectrum}

The SDSS spectrum of J1050+3456 is shown in Figure 1 and measurements are given in Table 1 . The spectrum shows strong, narrow emission lines including [O III], $\mathrm{H} \beta$, [Ne III], and [O II], and conspicuous stellar absorption lines. The [O III] redshift is $z_{[\mathrm{OIII}]}=0.2720$, and the other narrow emission lines as well as the stellar absorption lines are consistent within $\sim 100 \mathrm{~km} \mathrm{~s}^{-1}$. The intensity ratios among the narrow lines are typical of AGN. Relative to $z_{[\mathrm{O} \text { III] }}$, the broad $\mathrm{H} \beta$ line peaks at a blueshift of $3638 \pm 160 \mathrm{~km} \mathrm{~s}^{-1}$ and has a FWHM of $2170 \pm 250 \mathrm{~km} \mathrm{~s}^{-1}$. The peak of the broad $\mathrm{H} \alpha$ line shows a similar blueshift $\left(3470 \pm 150 \mathrm{~km} \mathrm{~s}^{-1}\right)$, but there is a more prominent red wing. (Values in Table 1 for $\mathrm{Mg}$ II and the [Ne v] flux and EW are from the Keck spectrum described below, with flux scaled to the SDSS spectrum using [Ne III]; other measurements are from the SDSS spectrum.) 


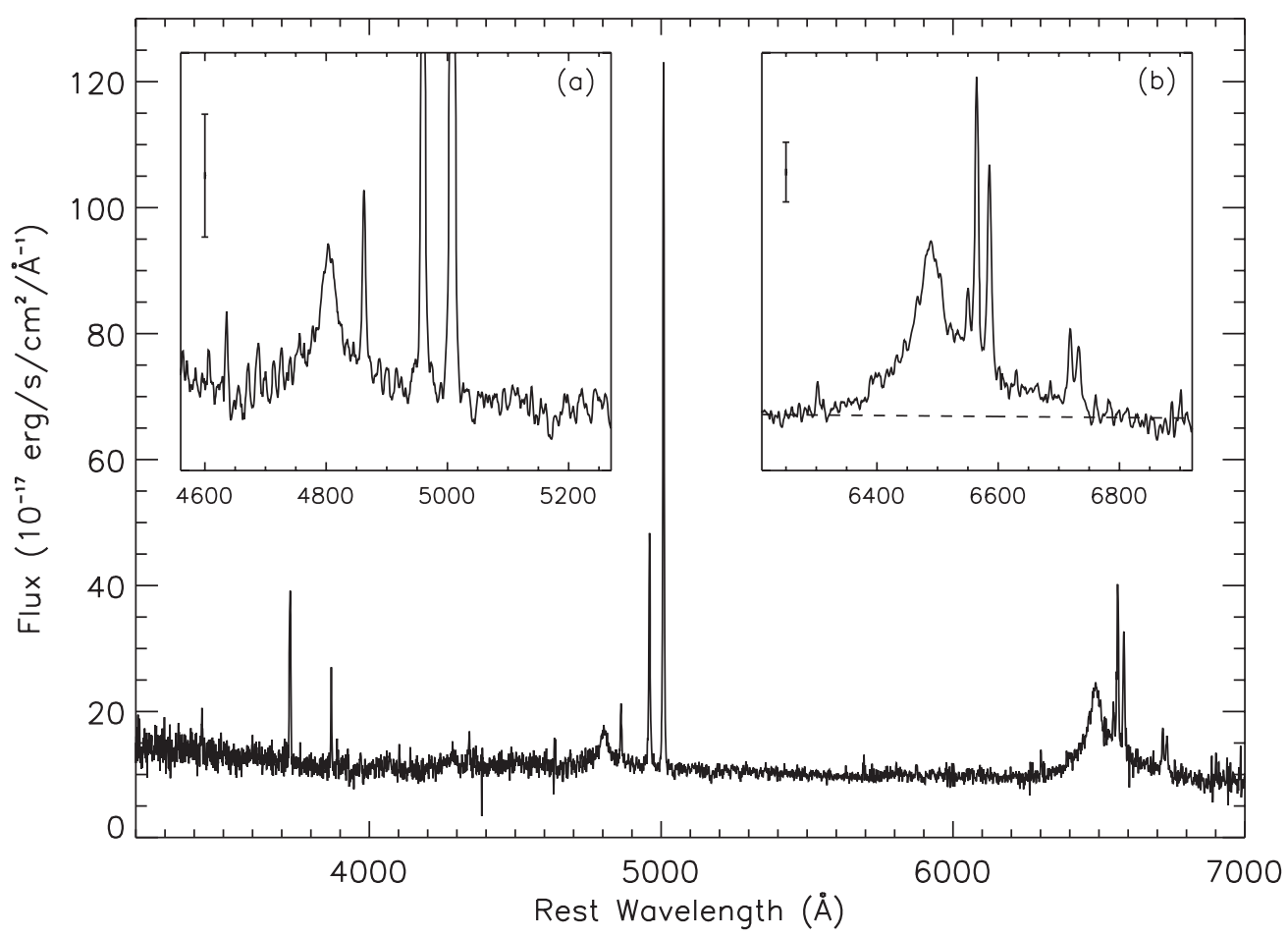

Figure 1. SDSS spectrum of J1050+3456 plotted as observed flux vs. rest wavelength. Insets show the regions of the $\mathrm{H} \beta$ (left) and $\mathrm{H} \alpha$ (right) lines. Note the blueshift of the peaks of the Balmer lines relative to the narrow lines, the symmetrical profile of $\mathrm{H} \beta$, and the extended red wing of $\mathrm{H} \alpha$. In both insets, the vertical bar represents $5 \times 10^{-17} \mathrm{erg} \mathrm{cm}^{-2} \mathrm{~s}^{-1} \AA^{-1}$.

Table 1

Emission-line Measurements

\begin{tabular}{|c|c|c|c|c|c|c|c|c|c|c|c|c|c|c|}
\hline Ion & {$[\mathrm{S}$ II] } & [S II] & {$[\mathrm{N}$ II] } & $\mathrm{H} \alpha(\mathrm{n})$ & $\mathrm{H} \alpha(\mathrm{b})$ & {$[\mathrm{OI}]$} & [O III] & {$[\mathrm{O} \mathrm{III}]$} & $\mathrm{H} \beta(\mathrm{n})$ & $\mathrm{H} \beta(\mathrm{b})$ & {$[\mathrm{Ne}$ III] } & [O II] & {$[\mathrm{Ne} \mathrm{v}]$} & $\operatorname{Mg}{ }_{\text {II }}(\mathrm{b})$ \\
\hline$\lambda_{\text {rest }}$ & 6730.8 & 6716.4 & 6583.4 & 6562.8 & 6562.8 & 6300.3 & 5006.8 & 4958.9 & 4861.4 & 4861.4 & 3869.1 & 3727.3 & 3425.9 & 2799.2 \\
\hline$\lambda_{\mathrm{obs}}$ & 8561.8 & 8544.6 & 8374.3 & 8348.1 & 8254.5 & 8013.4 & 6368.6 & 6307.6 & 6183.3 & 6109.1 & 4920.7 & 4741.4 & 4357.2 & 3522.8 \\
\hline$z$ & 0.2720 & 0.2722 & 0.2720 & 0.2720 & 0.2578 & 0.2719 & 0.2720 & 0.2720 & 0.2719 & 0.2570 & 0.2718 & 0.2720 & 0.2718 & 0.2585 \\
\hline Flux & 60 & 72 & 180 & 230 & 2200 & 20.3 & 760 & 270 & 63 & 700 & 70 & 220 & 35 & 520 \\
\hline Error & 6 & 7 & 18 & 12 & 300 & 5 & 38 & 13 & 6 & 70 & 4 & 11 & 7 & 80 \\
\hline EW & 5.4 & 6.5 & 12.1 & 15.5 & 230 & 2.0 & 71 & 24 & 5.9 & 66 & 6.5 & 19 & 2.6 & 47 \\
\hline
\end{tabular}

Notes. Emission-line measurements for J1050+3456; "b" and "'n" denote peaks of the "broad" and "narrow" components. Wavelength (air) and equivalent width are given in $\AA$; flux is in $10^{-17} \mathrm{erg} \mathrm{cm}^{-2} \mathrm{~s}^{-1}$. Flux and EW are in observed frame.

The observed continuum flux at $5100(1+z) \AA$ is $F_{\lambda}=$ $10.6 \times 10^{-17} \mathrm{erg} \mathrm{cm}^{-2} \mathrm{~s}^{-1} \AA^{-1}$. We have estimated the galaxy contribution to the continuum by modeling the observed spectrum using a set of empirical galaxy spectra from SDSS (Subbarao et al. 2002), combined with a simple power law with an adjustable spectral index for the AGN spectrum. The best fit is for an early-type galaxy. We estimate the galaxy fraction of the observed continuum at rest wavelength $5100 \AA$ to be approximately $36 \%$ with an uncertainty of 0.1 dex. For a typical early-type galaxy energy distribution, this implies an absolute magnitude $M_{B}=-20.0$ for the host galaxy. For the local black hole-bulge relationship (Tremaine et al. 2002, and references therein) the expected $M_{\mathrm{BH}}$ is $10^{8.2 \pm 0.3} M_{\odot}$, where the uncertainty reflects the scatter in the $M_{\mathrm{BH}}$-bulge luminosity relationship. Here, we have assumed that the observed stellar light is all bulge and made no allowance for fading.

The AGN component of the flux at $5100(1+z) \AA$ is $F_{\lambda}=7.4 \times$ $10^{-17} \mathrm{erg} \mathrm{cm}^{-2} \mathrm{~s}^{-1} \AA^{-1}$, giving $\lambda L_{\lambda}(5100)=10^{44.13} \mathrm{erg} \mathrm{s}^{-1}$. This implies a bolometric luminosity $L_{\text {bol }} \approx 9 \lambda L_{\lambda}(5100)=$ $10^{45.09} \mathrm{erg} \mathrm{s}^{-1}$, following Kaspi et al. (2000). Objects with shifted broad lines may not conform to the usual relationship between FWHM and black hole mass. However, if we apply Equation (2) of Shields et al. (2003), we find $M_{\mathrm{BH}}(\mathrm{H} \beta) \approx$ $10^{7.5} M_{\odot}$, using the measured $\mathrm{H} \beta$ FWHM of $2200 \mathrm{~km} \mathrm{~s}^{-1}$. For this $M_{\mathrm{BH}}$, the Eddington ratio of the AGN is $L / L_{\mathrm{Ed}}=10^{-0.5}$, a typical value.

The FIRST survey (Becker et al. 1995) detected no radio source at $20 \mathrm{~cm}$ at the position of $\mathrm{J} 1050+3456$ down to a limit of $1 \mathrm{mJy}$. This limit corresponds to a Kellermann et al. (1989) radio loudness parameter $\log R<0.5$, assuming a radio spectral index $F_{v} \propto v^{-0.5}$ between 6 and $20 \mathrm{~cm}$. Radio loud sources have $\log R>2$.

\subsection{Keck Spectra}

Blue and red spectra of J1050+3456 were obtained on the morning of 2008 December 24 with the Low Resolution Imaging Spectrometer (LRIS) double spectrograph on the Keck I telescope (Oke et al. 1995). On the blue side, the 400/3400 grism (spectral resolution of $7.2 \AA$ FWHM) was used, which covered a wavelength region from the atmospheric cutoff to the dichroic at $\sim 5500 \AA$. On the red side, the $600 / 7500$ grating 


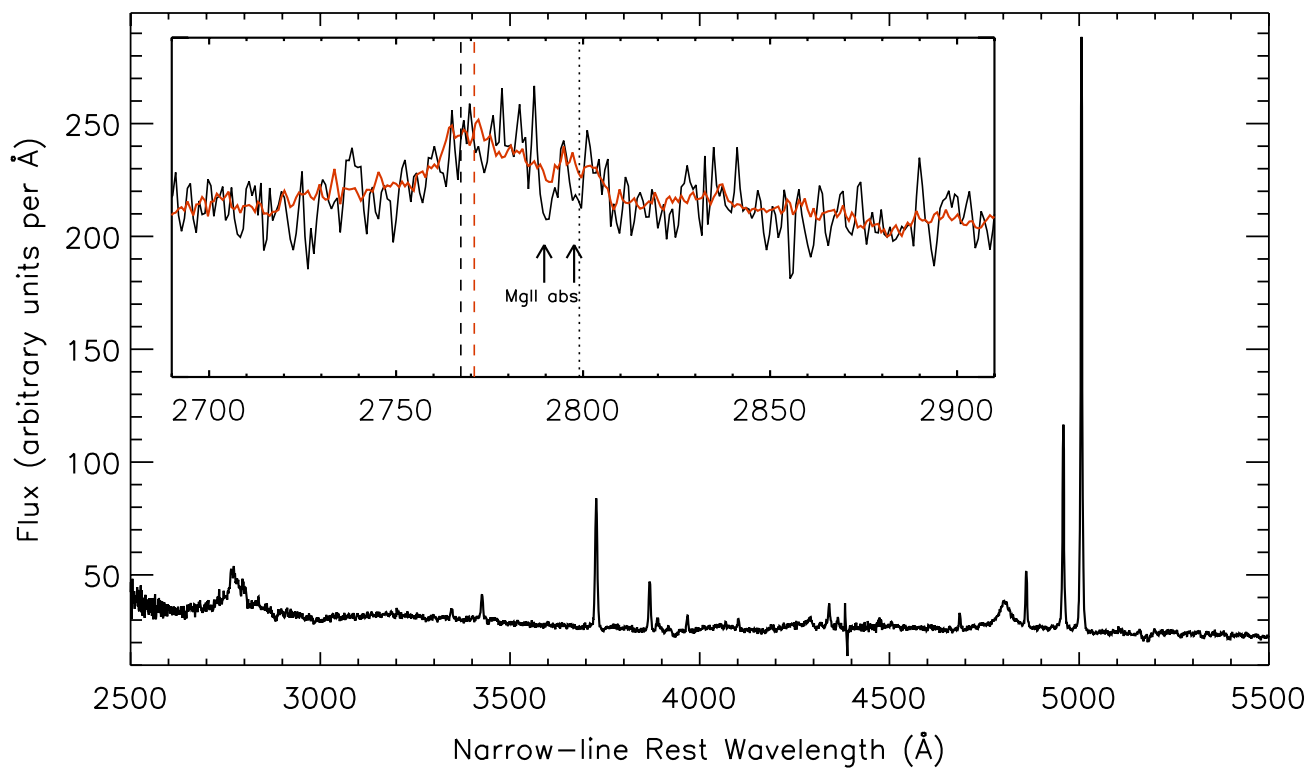

Figure 2. Keck LRIS spectrum of J1050+3456 combining the $400 \mathrm{~g}$ blue and $600 \mathrm{~g}$ red spectra (2008 December). Inset shows the region of the broad Mg II line, with the $400 \mathrm{~g}$ spectrum in red and the higher resolution $600 \mathrm{~g}$ spectrum in black. Arrows mark the $\mathrm{Mg}$ II doublet with a blueshift of $500 \mathrm{~km} \mathrm{~s}{ }^{-1}$. The black dashed line indicates the expected position of $\lambda 2800$ for the redshift of the $\mathrm{H} \beta$ broad line peak, while the red dashed line is the measured peak position of the Mg II broad line.

(A color version of this figure is available in the online journal.)

(spectral resolution of $4.7 \AA$ FWHM), centered at $6600 \AA$, covered a wavelength baseline of $2620 \AA$. The spectrum is shown in Figure 2 and measurements are given in Table 1.

One goal was to measure the wavelength and profile of the Mg II $\lambda \lambda 2796,2803$ line, noted by Bonning et al. (2007) as a test of black hole recoil in cases of shifted Balmer lines. The $\mathrm{Mg}$ II profile in Figure 2 is irregular and the central wavelength somewhat ambiguous. Accordingly, we obtained a second LRIS spectrum, this time with a blue side 600/4000 grism (spectral resolution of $4.0 \AA$ FWHM), on the evening of 2009 April 9. Though at much lower signal-to-noise ratio $(\mathrm{S} / \mathrm{N})$, the improved spectral resolution of the newer blue spectrum makes clear that the red wing of the broad $\mathrm{Mg}$ II feature is affected by a narrow Mg II absorption doublet at a blueshift of $500 \pm 50 \mathrm{~km} \mathrm{~s}^{-1}$ with respect to $z_{[\mathrm{O} \text { III] }}$. Because of the absorption doublet and limited $\mathrm{S} / \mathrm{N}$ of the spectra, the line peak and centroid velocity of $\mathrm{Mg}$ II remain uncertain. The $\mathrm{Mg}$ II peak in the $400 \mathrm{~g}$ spectrum falls at $\sim-3151 \mathrm{~km} \mathrm{~s}^{-1}$, which is $490 \mathrm{~km} \mathrm{~s}^{-1}$ less than the $\mathrm{H} \beta$ peak shift. If this peak velocity is adopted for $\mathrm{Mg}$ II, then the profile has a substantial red wing, more pronounced than $\mathrm{H} \alpha$. The $\mathrm{Mg}$ II centroid in the $400 \mathrm{~g}$ spectrum has a blueshift $\sim 2300 \pm 300 \mathrm{~km} \mathrm{~s}^{-1}$ relative to $z_{[\mathrm{O}}{ }_{\text {III] }}$, substantially less than the blueshift of the broad $\mathrm{H} \beta$ line.

A common pattern in normal AGN is for [Ne v] 33426 to be wider than $\left[\mathrm{O}_{\mathrm{III}}\right] \lambda 5007$. Bonning et al. (2007) suggest that this should not occur for an ionizing source displaced from the galactic nucleus by recoil. The SDSS spectrum lacks sufficient $\mathrm{S} / \mathrm{N}$ for [Ne v]. The 600/4000 LRIS spectrum shows a FWHM for [Ne v] of $5.4 \pm 0.3 \AA$, only slightly larger than the instrumental FWHM of $4 \AA$. Because of this marginal resolution, and the possibility that the QSO image did not completely fill the spectrograph slit, we derived the effective spectral resolution by forcing the corrected FWHM of [O II] 33727 in the $600 \mathrm{~g} \mathrm{~mm}^{-1}$ spectrum to agree with that of the SDSS spectrum, which has better spectral resolution. The result is a Keck instrumental FWHM of $3.6 \AA$, yielding a corrected FWHM for [Ne v] of $277 \pm 30 \mathrm{~km} \mathrm{~s}^{-1}$. This is close to the [O III] corrected FWHM of $273 \mathrm{~km} \mathrm{~s}^{-1}$ from the SDSS spectrum.

\section{THE NATURE OF J1050+3456}

\subsection{Superposition in a Cluster?}

Based on the SDSS field images around the QSO, as well as an examination of photometric redshifts of nearby bright galaxies, it is clear that $\mathrm{J} 1050+3456$ is not in a rich cluster environment. However, the nearest bright galaxy to the QSO does have a comparable photometric redshift, and has colors and a derived absolute magnitude $\left(M_{B} \sim-21.7\right)$ consistent with a massive early-type system. Since luminous red galaxies are generally found in relatively clustered environments (Zehavi et al. 2005), J1050+3456 could possibly reside within a sparse cluster or galaxy group. Deeper imaging and spectroscopy of faint galaxies around the QSO may resolve this issue.

In a discussion of J0927+2943, Shields et al. (2009) estimate a probability of order $10^{-4}$ of superposition within 1 arcsec of two AGNs with a velocity difference of order $2600 \mathrm{~km} \mathrm{~s}^{-1}$, representing a chance occurrence in a rich cluster of galaxies. Likewise, Heckman et al. (2009) draw the analogy between J0927+2943 and the radio galaxy NGC 1275 (Per A) at the center of the Perseus cluster of galaxies, which has two narrow emission-line systems offset in velocity by $\sim 3000 \mathrm{~km} \mathrm{~s}^{-1}$. $\mathrm{J} 1050+3456$ differs from J0927+2943 and NGC 1275 in that it does not have narrow emission lines at the peak velocity of the broad lines. AGNs rarely have a total absence of narrow lines at the systemic velocity. Weak narrow lines tend to occur when permitted Fe II is strong; however, Fe II is weak in $\mathrm{J} 1050+3456$. This argues against a superposition as the explanation of the spectrum of J1050+3456. A similar argument is given by Lauer \& Boroson (2009) for J1536+0441.

\subsection{A Supermassive Black Hole Binary?}

Supermassive black hole binaries are expected as galaxy mergers lead to coalescing galactic nuclei (Begelman et al. 1980). The orbital decay may stall at a radius of $1 \mathrm{pc}$, comparable to the size of the broad emission-line region (BLR). AGNs with shifted or double-peaked broad lines have long been discussed as candidates for binaries (Gaskell 1982), but the expected changes 
of velocity shift with orbital phase have not been observed. Recently, the binary interpretation has been offered for black hole recoil candidate J0927+2943 (Bogdanović et al. 2009; Dotti et al. 2009) and for the quasar SDSS J1536+0441 (Boroson \& Lauer 2008; Lauer \& Boroson 2009). Like J1050+3456, these systems have a broad $\mathrm{H} \beta$ peak blueshifted by a several thousand $\mathrm{km} \mathrm{s}^{-1}$. J0927+2943 has narrow lines at the velocity of the broad line peak, supporting the suggestion that it might represent a superposition of two AGNs (Heckman et al. 2009; Shields et al. 2009). For J1536+0441, a strong case can be made for a double-peaked emitter (Chornock et al. 2009; Gaskell 2009).

Is the binary interpretation viable for $\mathrm{J} 1050+3456$ ? The SDSS and Keck spectra together constrain the rate of change of the velocity shift of the $\mathrm{H} \beta$ broad line peak relative to the narrow lines, a stable reference. For the SDSS spectrum (dated 2005.17), the $\mathrm{H} \beta$ shift is $\Delta v=-3638 \pm 160 \mathrm{~km} \mathrm{~s}^{-1}$, relative to the redshift of $\left[\mathrm{O}_{\mathrm{III}}\right] \lambda 5007$, where the uncertainty is based on extreme cursor settings. For the Keck spectrum (2008.98), we find $\Delta v=-3546 \pm 120 \mathrm{~km} \mathrm{~s}^{-1}$. This gives a rate of change of $d v / d t=+31 \pm 60 \mathrm{~km} \mathrm{~s}^{-1}$ per rest-frame year. In the simple approximation that the AGN $\left(m_{2}\right)$ is in circular orbit around $m_{1}$ in a plane containing the observer, the rate of change of line-of-sight velocity $u_{2}$ is $d u_{2} / d t=$ $350 M_{1,8}^{-1} u_{2,3500}^{4} s_{\phi}^{4} c_{\phi}$ in $\mathrm{km} \mathrm{s}^{-1} \mathrm{yr}^{-1}$, where $M_{1,8} \equiv M_{1} / 10^{8} M_{\odot}$, $u_{2,3500} \equiv u_{2} / 3500 \mathrm{~km} \mathrm{~s}^{-1}$, and $s_{\phi}$ and $c_{\phi}$ are the sine and cosine of the azimuth $\phi$. For more detailed expressions (but with coefficients tailored to J0927+2943), see Bogdanović et al. (2009). If we interpret our measurement as an upper limit on $d u_{2} / d t$ of $70 \mathrm{~km} \mathrm{~s}^{-1} \mathrm{yr}^{-1}$ and take $m_{1}=10^{8.2} M_{\odot}$ and $m_{2}=10^{7.5} M_{\odot}$ (see Section 2.2), then $\phi$ is constrained to be within $\pm 15^{\circ}$ of quadrature, a fraction $17 \%$ of the entire orbit. For orbital inclination $i<90^{\circ}$ or for smaller masses, the constraint is tighter. The combined mass of the black holes cannot be much larger, if the normal $M_{\mathrm{BH}}$-bulge luminosity relationship applies.

\subsection{A Recoiling Black Hole?}

$\mathrm{J} 1050+3456$ is in some ways an interesting candidate for recoil. (1) The combination of large velocity shift and relatively narrow symmetrical profile, especially for $\mathrm{H} \beta$, is just the signature proposed by Bonning et al. (2007) for recoil candidates. (2) Our Keck spectrum shows a similar width for $[\mathrm{Ne} \mathrm{v}]$ and [O III], another criterion suggested by Bonning et al. (see discussion in Section 2.2). (3) Shields et al. (2009) suggested that the narrow emission lines of refractory elements might be weak in recoiling QSOs. A displaced ionizing source might preserve the order-of-magnitude depletions of Fe and other elements in the ISM, whereas these elements typically have normal gasphase abundances in the NLR (Nagao et al. 2003, and references therein). Nagao et al. give a mean value of I([Fe VII $] \lambda 6087) /$ $\mathrm{I}([\mathrm{Ne} \mathrm{V}] \lambda 3425)=0.52$ for type I AGNs (broad lines) and 0.34 for type II AGNs, whereas we find an upper limit of 0.12 in J1050+3456. Similarly, the composite Type II quasar spectrum of Zakamska et al. (2003) gives a value $\mathrm{I}(\mathrm{Mg}$ II $\lambda 2800)$ / $\mathrm{I}(\mathrm{H} \beta)=0.81$, whereas we find an upper limit of 0.16 for this ratio in the narrow line spectrum of $\mathrm{J} 1050+3456$. These results suggest that depletion of $\mathrm{Fe}$ and $\mathrm{Mg}$ into grains in the NLR of $\mathrm{J} 1050+3456$ may be more severe than in most AGNs.

On the other hand, there are reasons for caution. (1) $\mathrm{J} 1050+3456$ shows characteristics of a double peaked emitter (see below). (2) Shields et al. (2009) estimated a probability of only $\sim 10^{-4.9}$ for a line-of-sight kick velocity of $2650 \mathrm{~km} \mathrm{~s}^{-1}$ as observed for J0927+2943. For J1050+3456, the shift of $3500 \mathrm{~km} \mathrm{~s}^{-1}$ is closer to the theoretical maxi- mum of $4000 \mathrm{~km} \mathrm{~s}^{-1}$, resulting in an even lower probability. Note, however, that this argument is sensitive to the exact value of the maximum recoil velocity, currently based on extrapolation (Campanelli et al. 2007). Also note that Lauer \& Boroson (2009) argue that an ejection velocity of this magnitude is more likely to result from a three-body interaction than a gravitational radiation recoil event. (3) The [S II] doublet is marginally resolved with a typical line ratio of $\mathrm{I}(\lambda 6717) / \mathrm{I}(\lambda 6731)=$ $1.2 \pm 0.1$, implying an electron density $240 \pm 130 \mathrm{~cm}^{-3}$ from the "NEBULAR" software (Shaw \& Dufour 1995). This resembles the situation for the r-system narrow lines of J0927+2943, and the photoionization argument of Shields et al. (2009) carries over to $\mathrm{J} 1050+3456$ (given the similar [O II]/[O III] intensity ratio). With allowance for the electron density and continuum luminosity of J1050+3456, the result is a distance of $\sim 10^{0.3 \pm 0.2} \mathrm{kpc}$ for the ionizing source from the narrow emission-line gas. This indicates an age of $10^{5.5 \pm 0.2} \mathrm{yr}$, contributing to the low probability of catching a recoil in this stage. (4) The narrow-line spectrum of J1050+3456 is in most regards quite normal and conforms to the pattern of strong [O III] with weak Fe II. The question of whether the ionizing continuum from a displaced QSO would produce normal AGN narrow-line ratios in the host galaxy ISM requires investigation (Komossa et al. 2008).

\subsection{A Double-peaked Emitter?}

Double peaked broad Balmer lines are sometimes seen, and a number of cases have been interpreted in terms of line emission from a Keplerian disk (Eracleous \& Halpern 1994, and references therein). Occasionally, the broad Balmer lines may have a single peak shifted relative to the narrow lines. Often in such cases, the profile is asymmetric, with the red wing more extended in the case of a blueshifted peak, and vice versa, as discussed by Bonning et al. (2007). This pattern is evident in the $\mathrm{H} \alpha$ and $\mathrm{Mg}$ II profiles of J1050+3456 (Figures 1 and 2). In addition, the $M g_{I I}$ centroid is $2200 \mathrm{~km} \mathrm{~s}^{-1}$ blueshifted, smaller than for $\mathrm{H} \beta$. Bonning et al. (2007) noted a pattern for $\mathrm{Mg}$ II to have a smaller shift than $\mathrm{H} \beta$ among their displaced-peak AGNs. The $3500 \mathrm{~km} \mathrm{~s}^{-1}$ blueshift of the Balmer line peak of $\mathrm{J} 1050+3456$ is large but not unprecedented for a double-peaked emitter. In Table 3 of Strateva et al. (2003), among 138 double-peaked emitters, the shift of the blue peak $W_{\mathrm{b}}$ ranges from -6700 to $+800 \mathrm{~km} \mathrm{~s}^{-1}$, with 21 objects blueshifted $3500 \mathrm{~km} \mathrm{~s}^{-1}$ or more. However, the combination of the large shift and the dominance and symmetry of the blue peak of J1050+3456 is exceptional. In Strateva et al., FWHM for $\mathrm{H} \alpha$ ranges from 3700 to $20,700 \mathrm{~km} \mathrm{~s}^{-1}$; and the centroid at half-maximum, $\mathrm{FWHM}_{c}$, ranges from -2200 to $+1500 \mathrm{~km} \mathrm{~s}^{-1}$, with only 5 objects having $\mathrm{FWHM}_{c}$ more negative than $-1500 \mathrm{~km} \mathrm{~s}^{-1}$. The combination of low FWHM of $2200 \mathrm{~km} \mathrm{~s}^{-1}$ and large $\mathrm{FWHM}_{c}$ of $-3500 \mathrm{~km} \mathrm{~s}^{-1}$ for J1050+3456 stands apart among known double-peaked emitters. The reason for this is the weakness of the red wing and absence of a significant red peak, so that the width and centroid at half-maximum are determined by the blue peak alone.

Several studies have noted a tendency for the low ionization forbidden lines to be strong in double-peaked AGNs. J1050+3456 departs from this pattern. Eracleous \& Halpern (1994), working with radio loud objects, found that the ratio $\mathrm{I}\left(\left[\mathrm{O}_{\mathrm{I}}\right] \lambda 6300\right) / \mathrm{I}([\mathrm{O}$ III $] \lambda 5007)$ has a median value of 0.14 for their "disk-like emitters" but only 0.05 for other broad-line radio galaxies (BLRGs). From Table 1, we measure this ratio to be

\footnotetext{
http://stsdas.stsci.edu/nebular
} 
$0.03 \pm 0.01$, less than half the lowest value among the disklike emitters in Table 10 of Eracleous \& Halpern (1994).

The spectrum of J1050+3456 bears a strong resemblance to that of SDSS J1536+0441, originally suggested as a parsec scale binary black hole by Boroson \& Lauer (2008). J1536+0441 has a broad $\mathrm{H} \beta$ line peak blueshifted by $3500 \mathrm{~km} \mathrm{~s}^{-1}$, similar to the case of $\mathrm{J} 1050+3456$. Observations of $\mathrm{J} 1536+0441$ by Chornock et al. (2009) and Lauer \& Boroson (2009) make a strong case for a double-peaked emitter. Most significantly, the $\mathrm{H} \alpha$ broad line, out of range of the SDSS spectrum considered by Boroson \& Lauer (2008), shows a red peak at a similar velocity offset to the stronger blue peak. This example reinforces the case for caution in making exotic interpretations of J1050+3456 and other objects with displaced broad line peaks. The redblue asymmetry in $\mathrm{J} 1050+3456$ is more extreme than for $\mathrm{J} 1536+0441$. At a velocity of $v=+3500 \mathrm{~km} \mathrm{~s}^{-1}$ from the narrow line redshift (symmetrical with the blue peak), the SDSS spectrum of J1050+3456 shows a low red wing with little indication of a secondary peak or shoulder. The height of the red wing above the continuum at this velocity is $0.18 \pm 0.04$ times the height of the blue peak. The corresponding red/blue ratio in $\mathrm{J} 1536+0441$ is $0.43 \pm 0.04$. A further parallel between $\mathrm{J} 1050+3456$ and $\mathrm{J} 1536+0441$ is the presence of narrow $\mathrm{Mg}$ II absorption in both. In J1536+0441, the Mg II absorption is at $-240 \mathrm{~km} \mathrm{~s}^{-1}$ with respect to systemic velocity of the host galaxy, and in $\mathrm{J} 1050+3456$ it is at $-500 \mathrm{~km} \mathrm{~s}^{-1}$. Boroson \& Lauer (2008) argue that the geometry giving rise to the $\mathrm{Mg}$ II absorption may be difficult to explain in a model involving ejection of the black hole from the nucleus. For J1050+3456, we have estimated that the recoiling black hole is about $2 \mathrm{kpc}$ from the galactic center. It seems possible that material ejected from the nucleus at an earlier stage of the AGN, left over from a recent galactic merger, or associated with a companion galaxy, might cause the $\mathrm{Mg}$ II absorption.

An alternative model of $\mathrm{J} 1536+0441$, proposed by Tang \& Grindlay (2009), is a double-peaked emitter in a subparsec binary supermassive black hole system. In their picture, there is a normal, fairly symmetrical double peaked profile from a Keplerian disk around the more massive hole, and the blueshifted peak comes from another BLR associated with the secondary black hole. If such a model were applied to J1050+3456, the double-peaked disk emission would be considerably weaker relative to the blueshifted BLR emission from the secondary and would lack a well-defined peak on the red side of the line profile.

Could recoil (or three-body ejection) result in asymmetrical broad emission lines that mimic a double peaked emitter? The orbital period for the BLR is roughly $\sim 10^{2} \mathrm{yr}$, so there should be ample time for a normal, symmetrical BLR to be re-established in the $\sim 10^{5.5} \mathrm{yr}$ time since since the recoil event, as estimated above. This is the basis for rejection of asymmetrical broad line objects by Bonning et al. (2007). However, a better understanding of the dynamics of the retained accretion disk and BLR in a kicked AGN is needed, including the longevity of asymmetries from the initial transient and the effect of a different outer boundary condition for a wandering AGN disk.

\section{CONCLUSION}

J1050+3456 shows an unusual combination of a large velocity shift of the broad lines and a fairly symmetrical profile, especially for $\mathrm{H} \beta$. This object is in some respects an interesting candidate for black hole recoil, in particular its highly blueshifted yet symmetrical broad $\mathrm{H} \beta$ line and certain aspects of its narrow emission line spectrum. Nevertheless, the presence of a weak but visible red wing on $\mathrm{H} \alpha$, and the lesser blueshift of the $\mathrm{Mg}$ II line, suggest that this object is most likely an extreme member of the class of double-peaked emitters. Observations of the rest far-UV spectrum are needed to establish the velocity and profile of the high ionization broad lines, such as C IV, which tend to be narrower and less shifted than the Balmer lines in double-peaked emitters (Eracleous et al. 2004; Chornock et al. 2009). Improved observations of the Mg II line profile are needed for a definitive measurement of the peak velocity and profile, as well as the strength of narrow Mg II. High resolution imaging can test for displacement of the AGN from the galactic nucleus and for tidal features indicative of a recent merger, as might be expected if the object is a recoil event or binary. Multi-epoch spectroscopic monitoring can tighten constraints on the binary model and test for broad-line variability, as often occurs for double-peaked emitters. If $\mathrm{J} 1050+3456$ is confirmed as a double-peaked emitter, its extreme properties may lead to a better understanding of this class of AGNs and help to develop criteria for distinguishing true cases of recoil or binary supermassive black holes.

G.S. acknowledges support from the Jane and Roland Blumberg Centennial Professorship in Astronomy at the University of Texas at Austin. Funding for the Sloan Digital Sky Survey (SDSS) has been provided by the Alfred P. Sloan Foundation, the Participating Institutions, NASA, NSF, the U.S. Department of Energy, the Japanese Monbukagakusho, and the Max Planck Society. The W. M. Keck Observatory, which is operated as a scientific partnership among the California Institute of Technology, the University of California, and the National Aeronautics and Space Administration. The Observatory was made possible by the generous financial support of the W. M. Keck Foundation.

\section{REFERENCES}

Becker, R. H., White, R. L., \& Helfand, D. J. 1995, ApJ, 450, 559

Begelman, M. C., Blandford, R. D., \& Rees, M. J. 1980, Nature, 287, 307

Blecha, L., \& Loeb, A. 2008, MNRAS, 390, 1311

Bogdanović, Eracleous, M., \& Sigurdsson, S. 2009, ApJ, 697, 288

Bonning, E. W., Shields, G. A., \& Salviander, S. 2007, ApJ, 666, L13

Boroson, T., \& Lauer, T. 2008, Nature, 458, 53

Campanelli, M., Lousto, C., Zlochower, Y., \& Merritt, D. 2007, Phys. Rev. Lett., 98, 231102

Chornock, R., et al. 2009, arXiv:0907.0849

Dotti, M., Montuori, C., Decarli, R., Volonteri, M., Colpi, M., \& Haardt, F. 2009, MNRAS, 398, L73

Eracleous, M., \& Halpern, J. P. 1994, ApJS, 90, 1

Eracleous, M., et al. 2004, in IAU Symp. 222, The Interplay Among Black Holes, Stars, and ISM in Galactic Nuclei, ed. T. Storchi-Bergmann, L. C. Ho, \& H. R. Schmitt (Dordrecht: Kluwer), 29

Gaskell, C. M. 1982, ApJ, 263, 79

Gaskell, C. M. 2009, arXiv:0903.4447

Heckman, T. M., Krolik, J. H., Moran, S., Schnittman, J., \& Gezari, S. 2009, ApJ, 695,363

Kaspi, S., et al. 2000, ApJ, 533, 631

Kellermann, K. I., Sramek, R., Schmidt, M., Shaffer, D. B., \& Green, R. F. 1989, AJ, 98, 1195

Komossa, S., Zhou, H., \& Lu, H. 2008, ApJ, 678, L81

Lauer, T., \& Boroson, T. 2009, ApJ, 703, 930

Loeb, A. 2007, Phys. Rev. Lett., 99, 041103

Madau, P., \& Quataert, E. 2004, ApJ, 606, L17

Nagao, T., Murayama, T., Shioya, Y., \& Taniguchi, Y. 2003, AJ, 125, 1729 
Oke, J. B., et al. 1995, PASP, 107, 375

Shaw, R. A., \& Dufour, R. J. 1995, PASP, 107, 896

Shields, G. A., Bonning, E. W., \& Salviander, S. 2009, ApJ, 696, 1367

Shields, G. A., et al. 2003, ApJ, 583, 124

Smith, K. L., Shields, G. A., Bonning, E. W., \& Salviander, S. 2009, arXiv:0908.1998
Strateva, I. V., et al. 2003, AJ, 126, 1720

Subbarao, et al. 2002, Proc. SPIE, 4847, 452

Tang, S., \& Grindlay, J. 2009, ApJ, 704, 1189

Tremaine, S., et al. 2002, ApJ, 574, 740

Zakamska, N., et al. 2003, AJ, 126, 2125

Zehavi, I., et al. 2005, ApJ, 621, 22 